Results 431 cases enrolled in 44 centers in Japan since March 2012 to March 2016, and completed last follow-up on March 2017. Background data, age, gender, aneurysm size, dome/neck ratio, VER were not significant different in both arms, and mean and median length\% of used HGC was 68.6 and 69.9 in $\mathrm{H}$ Group and 1.2 and 0 in B Group. Primary endpoint in $\mathrm{H}$ group $(3.3 \%, 7 / 214)$ couldn't show significant difference $(\mathrm{p}=0.083)$ with that in $\mathrm{B}$ group (7.1\%, 15/211). However, evaluation by same definition with previously published report, showed major recanalization at 1 year after procedure in $\mathrm{H}$ group $(2.3 \%, 5 / 214)$ was significantly lower $(p=0.039)$ than B group $(6.6 \%, 14 /$ 211). And also, adverse event in $H$ group is significantly lower than that in B group and no aneurysm rupture in both groups.

Conclusion Second generation hydrogel coils decreases adverse outcomes including recanalization and unfavorable clinical events in endovascular treatment for intracranial aneurysms.

Disclosures N. Sakai: 1; C; Terumo/Microvntion, Medtronic, Neurovasc, Daiichi-Sankyo. 2; C; Asahi Intec, Biomedical Solutions, Johnson\&Johnson/Cerenovus, Medtronic, Neurovasc, Penumbra, Stryker, Terumo. H. Imamura: 2; C; Medtronic. C. Sakai: None. A. Hyodo: None. Y. Ito: None. Y. Matsumaru: None. S. Miyachi: None. S. Yoshimura: None. M. Sasaki: None. K. Ogasawara: None. S. Miyamoto: None. S. Miyamoto: None. M. Ezura: None. I. Nakahara: None. A. Ishii: None. T. Higashi: None.

\section{LB-008 MOTOR NEUROPROSTHESIS IMPLANTED USING CEREBRAL VENOGRAPHY IMPROVES ACTIVITIES OF DAILY LIVING IN SEVERE PARALYSIS}

${ }^{1} \mathrm{~T}$ Oxley, ${ }^{2} \mathrm{P}$ Mitchell ${ }^{*},{ }^{2} \mathrm{~N}$ Opie, ${ }^{2} \mathrm{P}$ Yoo, ${ }^{3} \mathrm{~J}$ Mocco, ${ }^{2} \mathrm{~B}$ Campbell, ${ }^{2} \mathrm{C}$ Bird, ${ }^{4} \mathrm{~S}$ Lee. ${ }^{1}$ University of Melbourne, Melbourne, Australia; ${ }^{2}$ Royal Melbourne Hospital, Melbourne, Australia; ${ }^{3}$ Mount Sinai Health System, New York City, NY; ${ }^{4}$ Calvary, Melbourne, Australia

\subsection{6/neurintsurg-2020-SNIS.280}

Background An implantable Brain Computer Interface (BCI) may function as a motor neuroprosthesis to restore voluntary motor function in patients with severe paralysis due to brain, spinal cord, peripheral nerve or muscle dysfunction, but to date demonstrations have been limited in clinical translation. We report the home-based use of a fully-implanted mobile motor neuroprosthesis using a novel endovascular stent-electrode, avoiding craniotomy, to restore voluntary motor control of a patient by means of a personal computer.

Methods The participant was a 75 -year-old man with flaccid paralysis in his upper limbs due to Motor Neuron Disease (MND). Prior to the study he was totally dependent on his caregiver wife for all Instrumental Activities of Daily Living (IADLs) including remote communication. Using routine cerebral venography techniques, the device was placed in the superior sagittal sinus adjacent to primary motor cortex and connected to a subcutaneous wireless transmitting unit. The participant underwent closed-loop training to convert
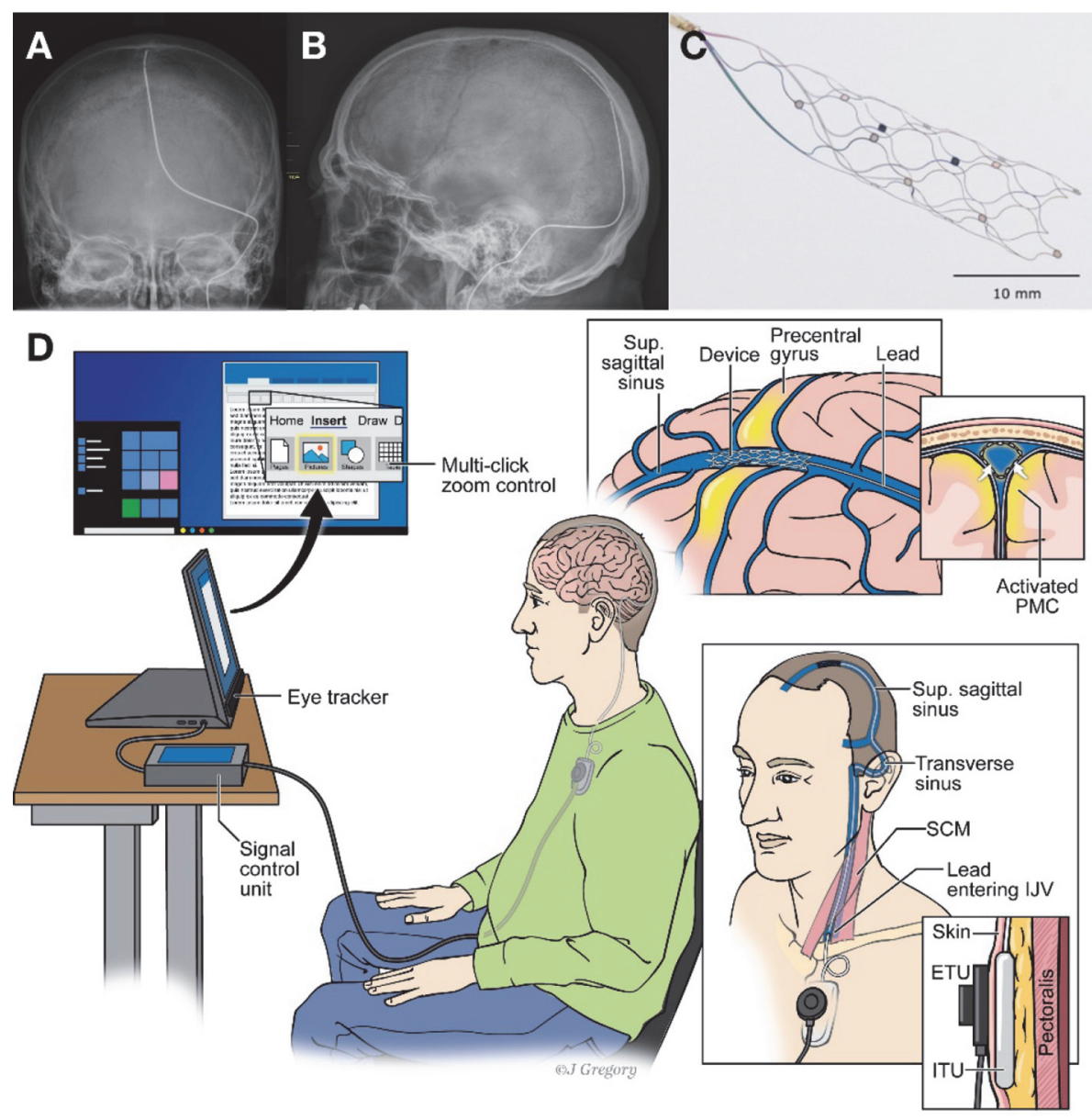

Abstract LB-008 Figure 1 Endovascular motor prosthesis system 
electrocorticographic activity from attempted movements into multiple mouse-click actions for click-selection, used in conjunction with an eye-tracker for cursor-navigation, to control a personal computer. System control performance was measured as click-selection accuracy during activities of daily living tasks including typing and emailing. Other metrics included the correct characters per minute typing speed, information transfer rate in bits/min of the motor prosthesis alone and system overall, improvement in capacity to perform IADLs. The presence of device-related thrombus on computerised tomography (CT) venography was assessed at 3 months.

Findings The participant was using the system at home and unsupervised on day 36 post system turn-on. During the typing task, a click-selection accuracy of $91 \cdot 0 \pm 4 \cdot 5 \%$ was achieved across 748 trials, at $12 \cdot 8 \pm 1 \cdot 1$ correct characters per minute with predictive text disabled. An information transfer rate of
$57 \cdot 3 \pm 5 \cdot 9 \mathrm{bits} / \mathrm{min}$ and $15 \cdot 1 \pm 2 \cdot 5 \mathrm{bits} / \mathrm{min}$ was achieved for the overall system and motor neuroprosthesis alone, respectively. He performed remote communication including messaging, shopping and managing his finances independently, resulting in a three-point increase in his Lawton IADL score. CT-venography study performed three months after implantation revealed no evidence of device migration, venous thrombosis or stenosis.

Interpretation A motor neuroprosthesis implanted via cerebral venography was used to restore voluntary motor function by way of computer control, which may represent a clinically feasible method of improving functional independence in patients with severe paralysis.

Disclosures T. Oxley: 4; C; Synchron. P. Mitchell: None. N. Opie: 4; C; Synchron. P. Yoo: 4; C; Synchron. J. Mocco: 4; C; Synchron. B. Campbell: None. C. Bird: None. S. Lee: None. 